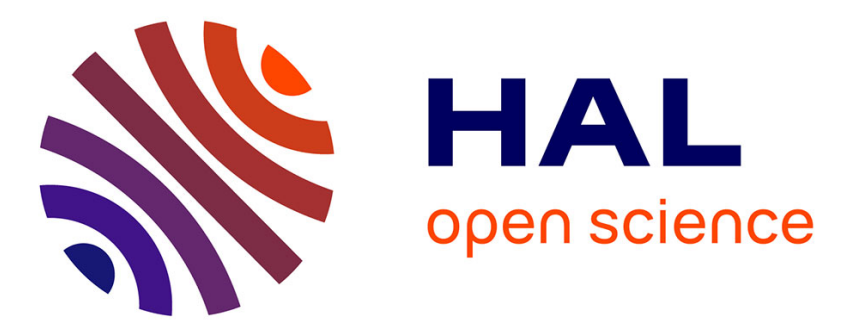

\title{
3D resistivity gridding of large AEM datasets: a step toward enhanced geological interpretation
}

\author{
Alexandre Pryet, James Ramm, Jean-Paul Chilès, Esben Auken, Benoît
}

Deffontaines, Sophie Violette

\section{- To cite this version:}

Alexandre Pryet, James Ramm, Jean-Paul Chilès, Esben Auken, Benoît Deffontaines, et al.. 3D resistivity gridding of large AEM datasets: a step toward enhanced geological interpretation. Journal of Applied Geophysics, 2011, 75, pp.277-283. 10.1016/j.jappgeo.2011.07.006 hal-00694658v2

\section{HAL Id: hal-00694658 \\ https://hal.science/hal-00694658v2}

Submitted on 8 May 2012

HAL is a multi-disciplinary open access archive for the deposit and dissemination of scientific research documents, whether they are published or not. The documents may come from teaching and research institutions in France or abroad, or from public or private research centers.
L'archive ouverte pluridisciplinaire $\mathbf{H A L}$, est destinée au dépôt et à la diffusion de documents scientifiques de niveau recherche, publiés ou non, émanant des établissements d'enseignement et de recherche français ou étrangers, des laboratoires publics ou privés. 


\title{
3D resistivity gridding of large AEM datasets: a step toward enhanced geological interpretation
}

\author{
Alexandre Pryeta, James Ramm ${ }^{\mathrm{b}}$, Jean-Paul Chilès ${ }^{\mathrm{c}}$, Esben Auken ${ }^{\mathrm{b}}$, \\ Benoit Deffontaines ${ }^{\mathrm{d}}$, Sophie Violette ${ }^{\mathrm{a}}$ \\ ${ }^{a}$ UPMC Univ. Paris 6 \& CNRS, UMR Sisyphe, 4 place Jussieu, 75252 Paris cedex 05, \\ France \\ ${ }^{b}$ University of Arhus, Department of Earth Sciences, HydroGeophysics Group, \\ Høegh-Gulbergs gade 2, 8000 Århus, Denmark \\ ${ }^{c}$ MINES ParisTech, Center of Geosciences and Geoengineering, Fontainebleau, France \\ ${ }^{d}$ UPE, GTMC Laboratory, Marne-La-Vallée, France
}

\begin{abstract}
We develop a technique allowing 3D gridding of large sets of $1 \mathrm{D}$ resistivity models obtained after inversion of extensive airborne EM surveys. The method is based on the assumption of a layered-earth model. 2D kriging is used for interpolation of geophysical model parameters and their corresponding uncertainties. The 3D grid is created from the interpolated data, its structure accurately follows the geophysical model, providing a lightweight file for a good rendering. Propagation of errors is tracked through the quantification of uncertainties from both inversion and interpolation procedures. The 3D grid is exported to a portable standard, which allows flexible visualization and volumetric computations, and improves interpretation. The method is validated and illustrated by a case-study on Santa Cruz Island, in the Galapagos Archipelago.

dx.doi.org/10.1016/j.jappgeo.2011.07.006
\end{abstract}

Keywords:

Airborne electromagnetics, 3D resistivity model, 3D grid, Kriging, Interpolation

Email address: a.pryet@gmail.com (Alexandre Pryet) 


\section{Introduction}

Airborne Electromagnetic (AEM) surveying can cover extensive areas in a short space of time, collecting thousands of soundings along hundreds of kilometers of flight lines. Numerous field surveys, based on frequency (FEM) and time-domain (TEM) electromagnetics have been successfully conducted in various complex environments and reported in literature (Bosch et al., 2009; Mogi et al., 2009; Steuer et al., 2009; Supper et al., 2009).

Recent advances to provide 3D imaging of the subsurface (Cox et al., 2010) are not widespread and may not significantly improve the quality of resistivity mapping (Viezzoli et al., 2010). As a consequence, most AEM datasets are inverted with a 1D model and are typically viewed as cross sections or 2D interpolated maps (e.g. Mullen and Kellett (2007); d'Ozouville et al. (2008a); Viezzoli et al. (2008). Some attempts are made to visualize the results in 3D (Bosch et al., 2009; Palamara et al., 2010), but without quantification of related uncertainties.

There is a need for an efficient and reliable methodology to visualize in 3D the structures identified by AEM surveys. To this end, it is important to understand under which assumptions, a 1D model description can reasonably resolve $2 \mathrm{D}$ and $3 \mathrm{D}$ structures.

An early paper on the subject (Newman et al., 1986) calculate the TEM response caused by 3D electromagnetic scattering and shows that the thickness of conductive overburdens and the depth to sedimentary layers beneath volcanic structures can be successfully resolved with 1D inversion. However, 3D conductors are often replaced by a conducting layer at similar depth and $1 \mathrm{D}$ inversion of 3D structures invariably results in non-unique models. Auken et al. (2008) studied the problem by calculating the EM forward response over theoretical $2 \mathrm{D} / 3 \mathrm{D}$ buried valley structures and inverted with a 1D laterally constrained least-squares inversion code Auken and Christiansen (2004). It is found that resistivities are well resolved when the slope of the dipping structures is below $30 \%$ and that resistivity contrasts are not much higher than 1:10. Advances in inversion techniques improve images of the subsurface and also offer, crucially, estimates of the model fit and resolution of model parameters. Spatially constrained inversion (Viezzoli et al., 2008), implements spatial constraints between models allowing the user to bias the outcome of the inversion to reflect the geological variability of the area. The effect of the constraints is that the model description is 3D with local 1D inversion kernels. It is clear that while 1D inversion does not produce flawless reconstructions of the subsurface, results over 3D struc- 
tures are acceptable when the structures are much larger than the footprint of the geophysical system (see Reid et al. (2006)).

The problem addressed in this paper is not the $3 \mathrm{D}$ visualization of the inversion results, but of accurately representing a scattered dataset of $1 \mathrm{D}$ models as a 3D grid. In turn, a 3D grid allows volumetric computations and is convenient to use for $3 \mathrm{D}$ visualization. In order to produce worthwhile and accurate images, two requirements shall be addressed by the gridding method:

1. At each 1D model location, the 3D grid should honor the model - i.e. resistivity and layer interfaces shall be preserved.

2. Away from the $1 \mathrm{D}$ models (between flight lines) the 3D grid of resistivity should be reliable enough to allow interpretation - i.e., uncertainties have to be quantified and should remain below some quality threshold.

After a presentation of the methodology to translate datasets of $1 \mathrm{D}$ models into a 3D grid of resistivity, we describe how to quantify the propagation of uncertainty from both the inversion and interpolation procedures. The method is validated on a case study in volcanic settings, the Galapagos Islands.

\section{Methodology}

The most straightforward technique to represent the resistivity in $3 \mathrm{D}$ from a collection of $1 \mathrm{D}$ vertical inversion models is to use $3 \mathrm{D}$ interpolation. Currently known 3D interpolation algorithms require discrete data in all directions, discarding the layered approach used in the inversion, and leading to a smoothing effect between previously defined layer boundaries. The other alternative, presented in this paper, is to interpolate the geophysical model parameters of the 1D models (layers resistivities and e.g. layer thicknesses) in the 2D horizontal space.

We start from the model vector $\mathbf{m}=\left(\mathbf{m}_{\mathbf{1}}, \ldots, \mathbf{m}_{\mathbf{N}}\right)^{T}$, a set of $N$ vertical resistivity models obtained after the inversion of $N$ soundings over the region of interest. Each vertical inversion model $\mathbf{m}_{\mathbf{i}}=\left(p_{i, 1}, \ldots, p_{i, 2 L}\right)^{T}$ is described by a vector of $2 L$ scalars $p_{i, k}$ describing the resistivity and geometry (thickness, or depth, or elevation) of the $L$ layers. Inversion models have the same number of layers throughout the study area. In some cases, the initial distribution of the geometry parameters is sufficiently close to 
normal so that transformation is not necessary before interpolation. Transformation of variables (e.g. by the logarithmic function) may be required before interpolation of some parameters, in particular resistivities. Note $\mathbf{m}_{\mathbf{i}}^{\prime}=\left(p_{i, 1}^{\prime}, \ldots, p_{i, 2 L}^{\prime}\right)^{T}$, the vector of transformed parameters of inversion model $i$ and $\mathbf{m}^{\prime}=\left(\mathbf{m}_{\mathbf{1}}^{\prime}, \ldots, \mathbf{m}_{\mathbf{N}}^{\prime}\right)^{T}$ the whole set of transformed inversion models.

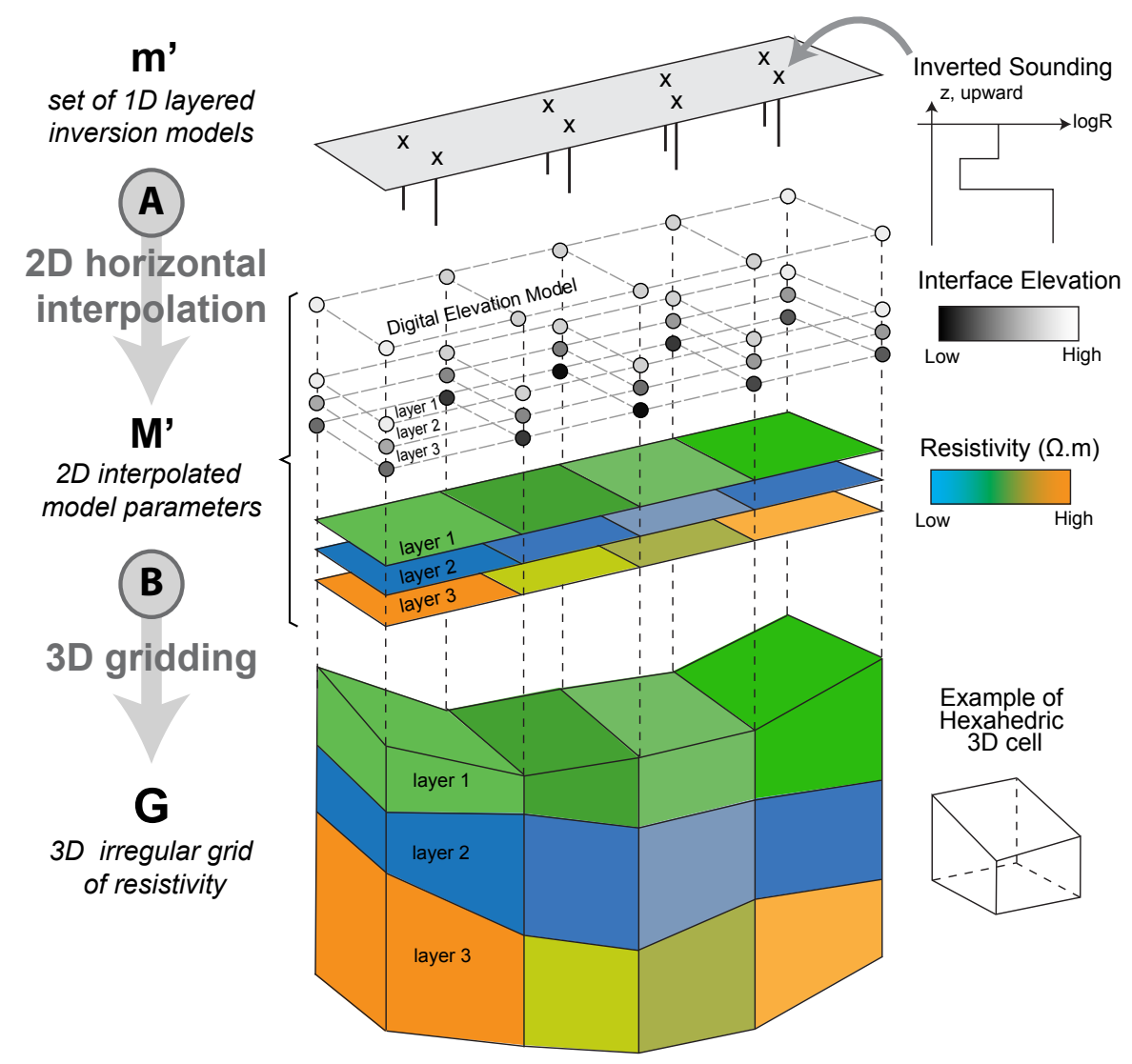

Figure 1: 3D gridding of resistivity (top to bottom). (A) From the set of $1 \mathrm{D}$ vertical inversion models, geophysical model parameters are interpolated in the $2 \mathrm{D}$ horizontal space. (B) The 3D grid is constructed from 3D vertex positions and filled with resistivity values from corresponding resistivity maps.

Layer thicknesses are not required in the case of "smooth" inversions with numerous layers whose thicknesses are fixed. To obtain a finite thickness of the 3D model, the thickness of the last layer (usually assumed to be infinite) is arbitrarily fixed to two or three times the thickness of the 
overlying layer.

The construction of the 3D grid of resistivity can be described by the succession of two operations.

First (Fig. 1, A), the $2 L$ transformed model parameters $p_{1}^{\prime}$, dots, $p_{2 L}^{\prime}$ in $\mathbf{m}^{\prime}$ are interpolated in the $2 \mathrm{D}$ horizontal space to matrices $\widehat{\mathbf{P}}_{\mathbf{1}}^{\prime}, \ldots, \widehat{\mathbf{P}}_{\mathbf{2} \mathbf{L}}^{\prime}$, whose size is equal to the number of cells discretizing the $2 \mathrm{D}$ domain. To obtain coherent 3D grids, the discretization must be the same for all parameters. These matrices are gathered into the $2 L$-vector $\widehat{\mathbf{M}}^{\prime}=\widehat{\mathbf{P}}_{\mathbf{1}}^{\prime}, \ldots, \widehat{\mathbf{P}}_{\mathbf{2} \mathbf{L}}^{\prime}$ containing all interpolated parameters (resistivity and geometry) of the layered resistivity model over the study area.

In a second phase (Fig. 1, B), the 3D log-resistivity field $\widehat{\mathrm{G}}^{\prime}$ is deduced from $\widehat{\mathbf{M}}^{\prime}$. It is represented by a $3 \mathrm{D}$ grid composed by hexahedral cells. The horizontal resolution of the grid is identical to the resolution of the 2D matrices. Vertically, there is one cell per geophysical layer so that the resolution of the grid follows the resolution of the resistivity model in that direction. The vertical coordinates, $z$, of the cell vertices are deduced from the digital elevation model (DEM) and the interpolated geometry parameter.

When dealing with 1D models where layer thicknesses vary, the choice of the geometry parameter to be interpolated (layer thickness, depth or elevation) has to be made with care. If the geometry of the geological formation is expected to follow the topography, layer thickness or interface depth is preferred, while interpolation of interface elevation should be chosen in other cases (Chilès and Delfiner, 2011).

The model parameters are interpolated by kriging. Among linear predictors, kriging is optimal in the sense that it minimizes the variance of the prediction error. Moreover, it provides a "prediction" or "kriging" variance $\sigma_{K R I}^{2}$ which quantifies the magnitude of the interpolation error. Kriging assumes that the spatial covariance or variogram of the parameters are known. An experimental variogram has to be computed for each parameter and variogram models have to be fitted.

Kriging requires no special assumption relative to the distribution of the studied parameter. Nevertheless, when the spatial distribution of the parameter is Gaussian, kriging provides the best linear unbiased estimator. Therefore it is recommended to apply kriging to variables whose histogram is not too far from a normal distribution (Chilès and Delfiner, 2011). Resistivity often has a lognormal distribution and is therefore transformed into its logarithm. In contrast, the parameter describing the geometry is often not too far from normality. 
Interpolation is performed by kriging with the Gstat package (Pebesma and Wesseling, 1998). The search radius shall be chosen to be larger than the spacing between flight lines in order to obtain a continuous 3D model. However, extending it to an unreasonable distance would slow the kriging algorithm without significant improvement. The resolution of the $2 \mathrm{D}$ grids must be adjusted depending on the variability of model parameters, the expected precision, and the acceptable computation time. Values of resistivity are predicted as "block" values, which allows the prediction of averaged values in the cell (Pebesma, 2001). Since the 3D cells of the grid are defined by their vertices, the parameters of the 3D grid (layer thickness, depth, or elevation) are also interpolated at cell vertices.

\section{Uncertainties and validation}

\subsection{Management of uncertainties}

Two sources of error affect the quality of the 3D grid of resistivity: the uncertainty on model parameters estimated by geophysical inversion, and the uncertainty due to the interpolation.

The inversion uncertainty, if provided by the inversion code, can be incorporated into the grid together with resistivity. Geophysical inversion based on a least-squares criterion provides an estimation of the uncertainty on estimated parameters from the linearized approximation to the covariance of the estimation error (Auken and Christiansen, 2004). The magnitude of the inversion uncertainty on $p_{i, k}$ the $k$-th model parameter at inversion model $i$ is quantified by the standard deviations $\sigma_{I N V \mid i, k}$. The $1 \mathrm{D}$ model at sounding $i$ is extended to $\mathbf{m}_{\mathbf{i}}=\left(p_{i, 1}, \ldots, p_{i, 2 L}, \sigma_{I N V \mid i, 1}, \ldots, \sigma_{I N V \mid i, 2 L}\right)^{T}$ - The inversion uncertainty can be due to poor signal quality during the sounding procedure or a lack of compatibility between the proposed geophysical model and the measured data during the inversion procedure (Auken and Christiansen, 2004). In spatially constrained inversion, an additional source of uncertainty may occur when a sounding is closely surrounded by others, with significant contrast in the signal. These sources of uncertainty are often likely to be spatially correlated and as a consequence, the standard deviations should be propagated by interpolation. Similarly to the inversion model parameters pk, the inversion standard deviations $\sigma_{I N V \mid k}$ can be interpolated by kriging, leading to matrices $\hat{\mathbf{S}}_{I N V \mid 1}, \ldots, \hat{\mathbf{S}}_{I N V \mid 2 L}$ in the $2 \mathrm{D}$ horizontal space.

The magnitude of the uncertainty due to the interpolation of model parameters $p_{k}$ is characterized by the kriging standard deviations matri- 
ces $\hat{\mathbf{S}}_{K R I \mid 1}, \ldots, \hat{\mathbf{S}}_{K R I \mid 2 L}$. These $2 \mathrm{~L}$ matrices are obtained as by-products of the kriging of the parameters $p_{k}$. As explained previously, the inversion variance $\sigma_{I N V \mid k}^{2}(x, y)$ depends on the quality of the soundings and the success of the inversion, while the kriging variance $\sigma_{K R I \mid k}^{2}(x, y)$ depends on the spatial variability of the parameter and the distance to data points. As a result, the two variances are considered as independent and can be summed to form the total uncertainty variance on model parameter $p_{k}$, defined as $\hat{\sigma}_{T O T, k}^{2}(x, y)=\hat{\sigma}_{I N V, k}^{2}(x, y)+\hat{\sigma}_{K R I, k}^{2}(x, y)$. If uncertainties from inversion are handled, the vector of 2D matrices of interpolated model parameters are therefore extended to $\widehat{\mathbf{M}}=\widehat{\mathbf{P}}_{1}, \ldots, \widehat{\mathbf{P}}_{2 L}, \ldots, \widehat{S}_{1}, \ldots, \widehat{\mathbf{S}}_{2 L}$ with total standard deviation matrices defined by Eq. 1. This leads to the construction of a 3D grid containing not only resistivity values but also related uncertainty.

$$
\widehat{\mathbf{S}}_{T O T, k}^{\prime}=\sqrt{\widehat{\mathbf{S}}_{I N V, k}^{\prime 2}+\hat{\mathbf{S}}_{K R I, k}^{\prime 2}}
$$

To facilitate interpretation, log-transformed parameters such as resistivities are back-transformed by exponentiation (in the Gaussian case, they are therefore median estimators). The related uncertainties are expressed by the Standard Deviation Factors (STDF) obtained by exponentiation of the log-resistivity total standard deviations. For parameter pk at location $(\mathrm{x}, \mathrm{y})$ of the discretized 2D space, the standard deviation factor is obtained from Eq. 2:

$$
S T D F_{k}(x, y)=\exp \left(\sigma_{T O T \mid k}(x, y)\right)
$$

Under the assumption that the error on log-resistivity is Gaussian and independent of the kriged estimate, the $(1-\alpha)$ confidence interval can be inferred with Eq. 3, where $\hat{p}_{k}(x, y)=\exp \left(\hat{p}_{k}^{\prime}(x, y)\right)$ is the back-transformed estimate $\hat{p}_{k}^{\prime}(x, y)$ and $z_{\beta}$ is the normalized Gaussian value corresponding to the cumulative probability $(1-\alpha / 2)$. With $z_{\beta}=1$, we obtain the $68 \%$ confidence interval.

$$
\frac{\hat{p}_{k}(x, y)}{\operatorname{STDF}_{k}(x, y)^{z_{\beta}}} \leq p_{k}(x, y) \leq \hat{p}_{k}(x, y) \cdot \operatorname{STDF}_{k}(x, y)^{z_{\beta}}
$$

The confidence intervals of parameters that have not been transformed (e.g. layer thicknesses) can be derived directly from the total variances. These confidence intervals are however approximations because thicknesses are not exactly Gaussian. 


\subsection{Validation of the $3 D$ grid}

Two aspects deserve to be addressed in order to validate the $3 \mathrm{D}$ resistivity grid. Before kriging, the applicability of the interpolation method to the given data set must be investigated. After kriging, the consistence of the interpolated parameters and inversion models should be estimated.

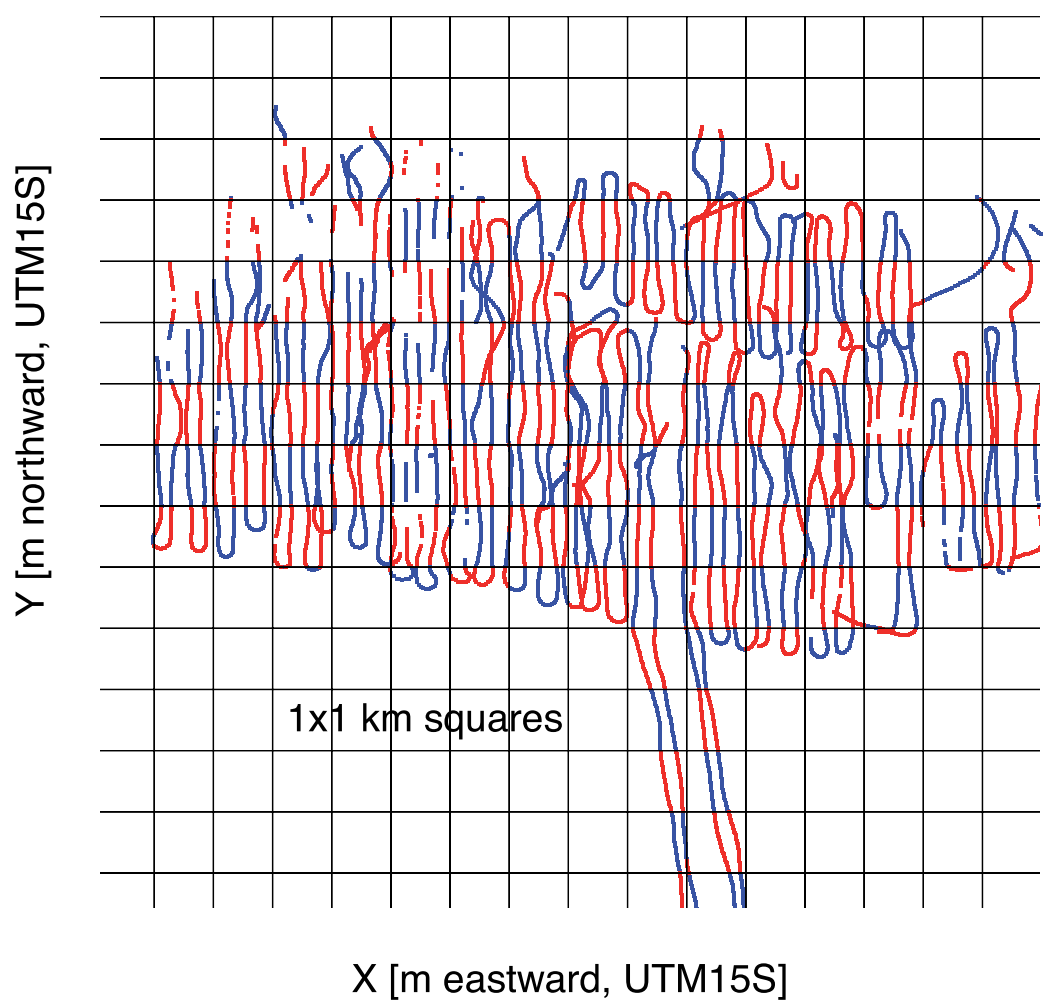

Figure 2: Validation of the prediction method using data split into two subsets S1 (blue) and S2 (red). Values at locations of S2 are predicted from S1. Example from SkyTEM data set collected by d'Ozouville et al. (2008a).

In order to validate the prediction method, the collection of 1D models is split into two subsets S1 and S2 similar to the white and black squares of a chessboard (Fig. 2). Parameters of inversion models falling within the "black" squares (within S2) are predicted from values falling within the "white" squares (within S1). For the method to be relevant, artificial gaps of data have to be at least as big as natural gaps, determined by spacing 
between flight lines. At inversion model $i$ in S2, the misfit between estimated values and original model parameters, the prediction error e(i), is obtained from Eq. 4.

$$
e(i)=\hat{p}_{k}^{\prime}\left(x_{i}, y_{i}\right)-p_{k}^{\prime}(i)
$$

where $p_{k}^{\prime}(i)$ is the $k$-th parameter of the $i$-th inversion model, and $\hat{p}_{k}^{\prime}(i)$ the estimate at model location $\left(x_{i}, y_{i}\right)$. If the statistical model is compatible with the dataset and the variogram model fits the experimental model, errors calculated on S2 should have a zero mean and for variance the kriging variance sigma ${ }_{K R I}^{2}$ (Chilès and Delfiner, 2011). Moreover, interpolated model parameters values should be found at $68 \%$ probability within the interval $\pm \sigma_{\hat{K} R I}$ from the estimates. As an additional precaution, it is recommended to repeat the operation switching S1 and S2. Under those conditions, kriging is considered applicable to the dataset, and predicted values $\hat{p}_{k}^{\prime}(i)$ will be provided with the confidence interval $\pm \sigma_{K R I}\left(x_{i}, y_{i}\right)$ at $68 \%$. After kriging with all data (S1 and S2), the fit between interpolated parameters and the inversion models should finally be estimated. If the validation step was successful and the horizontal resolution of the grid sufficiently fine, the fit between 2D matrix of interpolated parameter $p_{k}$ and original 1D models is expected to be good. It can be assessed with the root mean square error (RMSE) expressed in Eq. 5 where $p_{k}(i)$ stands for the value of parameter $k$ at the i-th 1D inversion model and the estimate of parameter pk at location $\left(x_{i}, y_{i}\right)$ of the $2 \mathrm{D}$ discretized horizontal space, the closest to the $i$-th $1 \mathrm{D}$ inversion model.

$$
\operatorname{RMSE}\left(p_{k}\right)=\sqrt{\frac{1}{N} \sum_{i=1}^{N}\left(\hat{p}_{k}\left(x_{i}, y_{i}\right)-p_{k}(i)\right)^{2}}
$$

\section{Case study: Galapagos Islands, Ecuador}

\subsection{Presentation of the survey}

In the frame of the project Galapagos Islands Integrated Water Studies (GIIWS) a large variety of investigations are under progress on the main inhabited island of Galapagos Archipelago, Santa Cruz. This basaltic island, whose last significant shield-building phase has been dated to approx. 500 000 y.b.p (Bow, 1979) is arid with the exception of the highlands. Rapid population growth rates have promoted the use of expensive desalination 
techniques while the lack of a sewage system leads to high contamination levels in the basal aquifer. There is an obvious need for a better understanding of hydrogeological processes on the island. To this end, an extensive SkyTEM survey has been conducted on the southern windward side of Santa Cruz (Fig. 3). Thousands of soundings have been collected along the $500 \mathrm{~km}$ of flight lines. Due to the nature of airborne surveys, the distribution of soundings is inhomogeneous, with a high density of soundings along flight lines (one sounding every 10 meters), and gaps of data in between (usually 250 meters wide). Results revealed interesting buried low-resistivity bodies in Santa Cruz, presented by d'Ozouville et al. (2008a) and Auken et al. (2009).

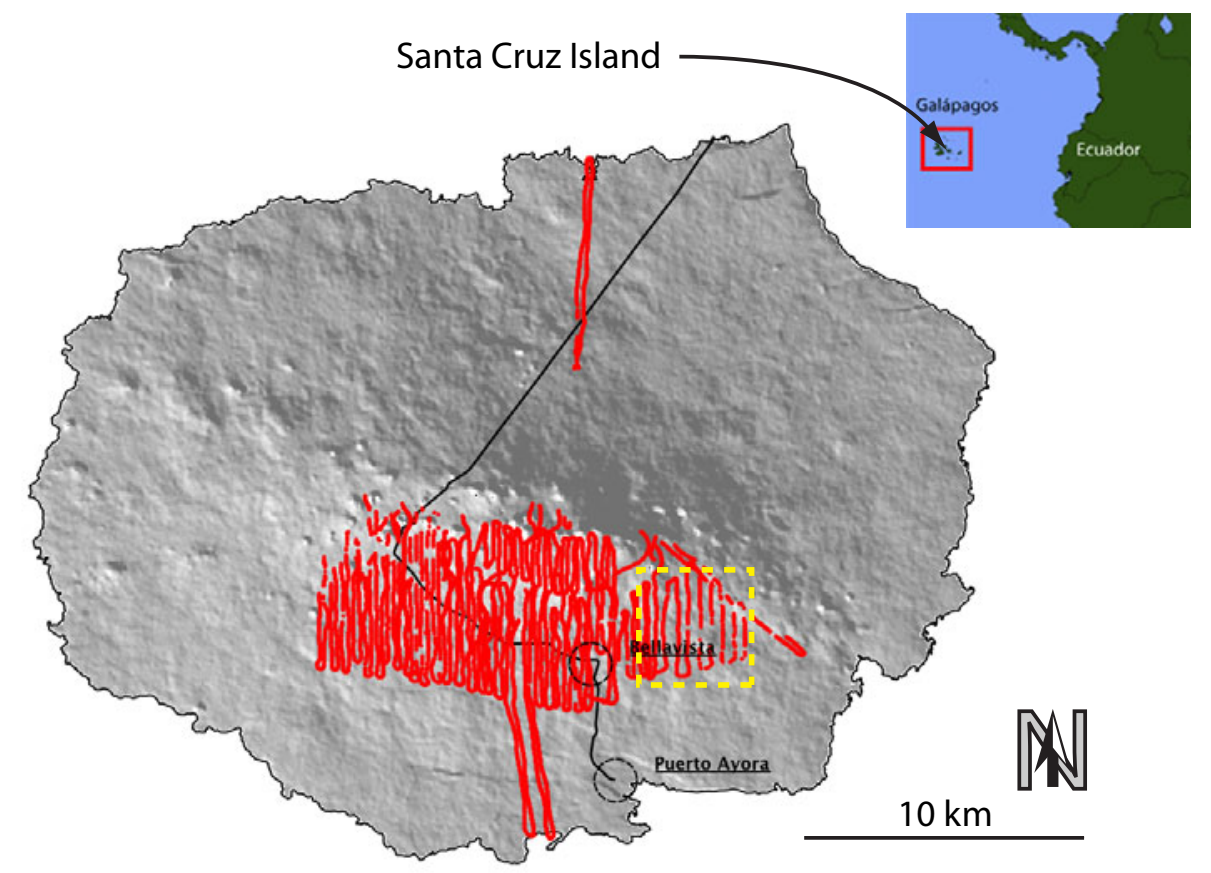

Figure 3: Location of SkyTEM survey: Santa Cruz Island, Galapagos Archipelago. Red dots show the flight lines, yellow box shows the data extent in Fig. 6

The soundings have been newly processed and inverted using the Spatially Constrained Inversion scheme (SCI) (Viezzoli et al., 2008) to a 19 layer "smooth" resistivity model, where the layer thicknesses are distributed logarithmically from the surface down to $250 \mathrm{~m}$ below topography. While the use of a spatially constrained inversion scheme is not compulsory, it provides 


\begin{tabular}{ccccccc}
\hline Layer & \multicolumn{3}{c}{ Variogram model of $\log (R)$} & \multicolumn{3}{c}{ Variogram model of $\sigma_{I N V}$} \\
\cline { 2 - 7 } & \multicolumn{3}{c}{ Bessel model } & \multicolumn{3}{c}{ Exponential model } \\
\cline { 2 - 7 } & $C_{N}$ & $a[\mathrm{~m}]$ & $C_{F}$ & $C_{N}$ & $a[\mathrm{~m}]$ & $C_{F}$ \\
\hline 1 & 0 & 92.6 & $3.28 \mathrm{E}-01$ & $3.05 \mathrm{E}-04$ & 7.3 & $4.15 \mathrm{E}-03$ \\
2 & 0 & 87.8 & $2.81 \mathrm{E}-01$ & $5.26 \mathrm{E}-04$ & 6.9 & $3.40 \mathrm{E}-03$ \\
3 & 0 & 87.3 & $2.42 \mathrm{E}-01$ & $5.10 \mathrm{E}-04$ & 6.6 & $3.46 \mathrm{E}-03$ \\
4 & 0 & 106.6 & $2.64 \mathrm{E}-01$ & $2.50 \mathrm{E}-04$ & 6.7 & $2.85 \mathrm{E}-03$ \\
5 & 0 & 107.6 & $2.82 \mathrm{E}-01$ & $2.91 \mathrm{E}-04$ & 6.8 & $2.77 \mathrm{E}-03$ \\
6 & 0 & 105.0 & $2.92 \mathrm{E}-01$ & $2.56 \mathrm{E}-04$ & 6.9 & $2.77 \mathrm{E}-03$ \\
7 & 0 & 131.5 & $3.60 \mathrm{E}-01$ & $2.86 \mathrm{E}-06$ & 6.8 & $2.11 \mathrm{E}-03$ \\
8 & 0 & 172.9 & $5.61 \mathrm{E}-01$ & $2.45 \mathrm{E}-05$ & 6.9 & $2.08 \mathrm{E}-03$ \\
9 & 0 & 164.5 & $5.38 \mathrm{E}-01$ & $3.79 \mathrm{E}-05$ & 6.9 & $2.06 \mathrm{E}-03$ \\
10 & 0 & 163.5 & $5.10 \mathrm{E}-01$ & $3.58 \mathrm{E}-05$ & 6.8 & $2.02 \mathrm{E}-03$ \\
11 & 0 & 159.1 & $5.00 \mathrm{E}-01$ & $2.59 \mathrm{E}-05$ & 6.8 & $2.01 \mathrm{E}-03$ \\
12 & 0 & 152.5 & $4.91 \mathrm{E}-01$ & $0.00 \mathrm{E}+00$ & 7.1 & $1.56 \mathrm{E}-03$ \\
13 & 0 & 127.5 & $4.15 \mathrm{E}-01$ & $0.00 \mathrm{E}+00$ & 7.1 & $1.55 \mathrm{E}-03$ \\
14 & 0 & 114.3 & $3.85 \mathrm{E}-01$ & $0.00 \mathrm{E}+00$ & 7.5 & $1.09 \mathrm{E}-03$ \\
15 & 0 & 100.4 & $4.09 \mathrm{E}-01$ & $0.00 \mathrm{E}+00$ & 7.5 & $1.07 \mathrm{E}-03$ \\
16 & 0 & 94.1 & $5.57 \mathrm{E}-01$ & $9.11 \mathrm{E}-06$ & 7.6 & $1.06 \mathrm{E}-03$ \\
17 & 0 & 97.5 & $7.51 \mathrm{E}-01$ & $3.16 \mathrm{E}-05$ & 9.7 & $2.92 \mathrm{E}-04$ \\
18 & 0 & 80.0 & $8.85 \mathrm{E}-01$ & $3.34 \mathrm{E}-05$ & 10.0 & $2.89 \mathrm{E}-04$ \\
19 & 0 & 68.8 & $1.36 \mathrm{E}+00$ & $4.67 \mathrm{E}-05$ & 10.9 & $2.84 \mathrm{E}-04$ \\
\hline
\end{tabular}

Table 1: Variogram model parameters of the log-transformed resistivity $(R,[\mathrm{ohm} . \mathrm{m}])$ fitted to 1st order K-Bessel model, and $\sigma_{I N V}$ fitted to exponential model for the 19 layers of the Santa Cruz SkyTEM dataset. $C_{N}$ is the nugget, $a$ the scale parameter, and $C_{F}$ the variance of the continuous component.

more consistent sets of neighboring models, and leads to the construction of more coherent 3D grids of resistivity.

For each layer, experimental variograms are computed for the log-transformed resistivity and the standard deviation from inversion. Fig. 4 presents the two variograms used for the first layer. The parameters of the variogram models for all the 19 layers are gathered in Table 1. The variograms of log-transformed resistivity fit well to isotropic Matérn models (also known as K-Bessel models) with shape parameter $\nu=1$ (Chilès and Delfiner, 2011; Pebesma and Wesseling, 1998). The variogram model reads $\gamma(h)=C_{N}+C_{F}\left(1-\frac{h}{a} K_{1}\left(\frac{h}{a}\right)\right)$ where $C_{N}$ is the nugget effect, $K_{1}$ is the modified Bessel function of the second kind of order $\nu=1, a$ and $C_{F}$ are the model parameters, and $h$ is the distance. The variograms of standard 

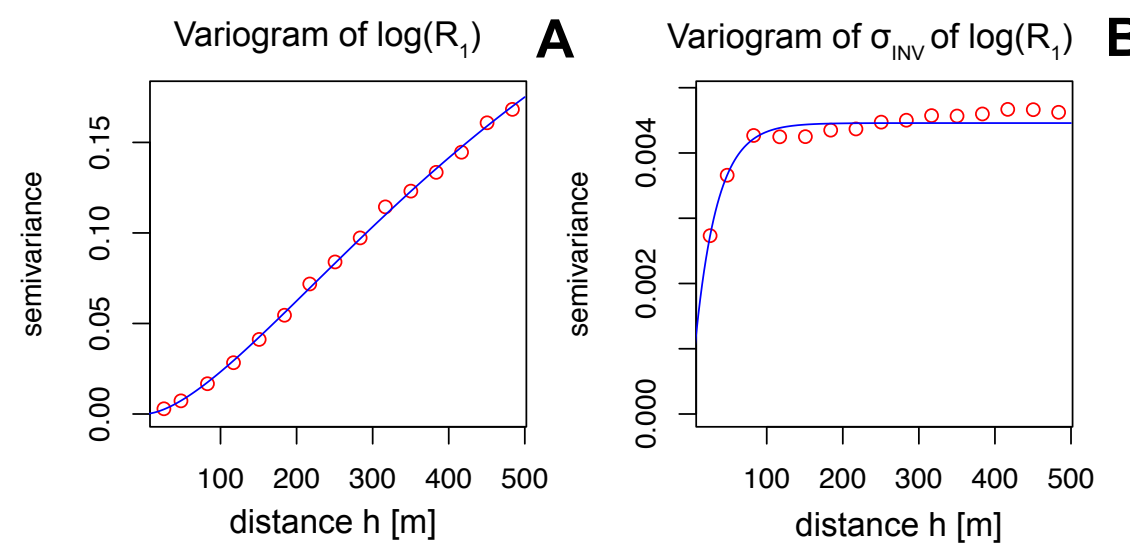

Figure 4: Sample variograms (red dots) and models (blue lines) used for the interpolation of the first layer. (A) For the log-transformed resistivity $\log (R)$, a first order Matérn or K-Bessel model $\left(\nu=1, a=92 \mathrm{~m}, C_{F}=0.33, C_{N}=0\right)$ fits well to the data. (B) For the standard deviation from inversion $\sigma_{I N V}$, an exponential model is more appropriate $(a=$ $\left.7.28 \mathrm{~m}, C_{F}=4.15 \times 10^{-3}, C_{N}=3.05 \times 10^{-4}\right)$.

deviation from inversion $\sigma_{I N V}$ fits better to the isotropic exponential model defined by (Pebesma and Wesseling, 1998). As presented in Table 1, the variograms of log-transformed resistivity, do not present a nugget effect $\left(C_{N}=0\right)$, and the sill $\left(C_{F}\right.$ in this case) increases for deeper layers. This is interpreted as the consequence of the sharp resistivity contrasts induce by sea water intrusion. The variograms of standard deviation from inversion $\sigma_{I N V}$ present a nugget effect, which represent the random component of inversion error. For deeper layers, a rise of the scale parameter $a$ is observed, while $C_{F}$ and $C_{N}$ decrease. This corresponds to a smaller sill and a larger range and reflects that for deeper layers, $\sigma_{I N V}$ is less variable and more spatially correlated.

\subsection{Validation of the method}

Before interpolation of parameters and construction of the 3D model, the applicability of the prediction method was investigated for the Santa Cruz survey. As described in section 3.2, the data set was split into two halves, S1 and S2, with a $1 \mathrm{~km}$-resolution regular grid (Fig. 2). Parameter values at locations of $\mathrm{S} 2$ were predicted from the parameter values in the subset S1.

As depicted in Fig. 5 (A), the misfit of predicted values of resistivi- 

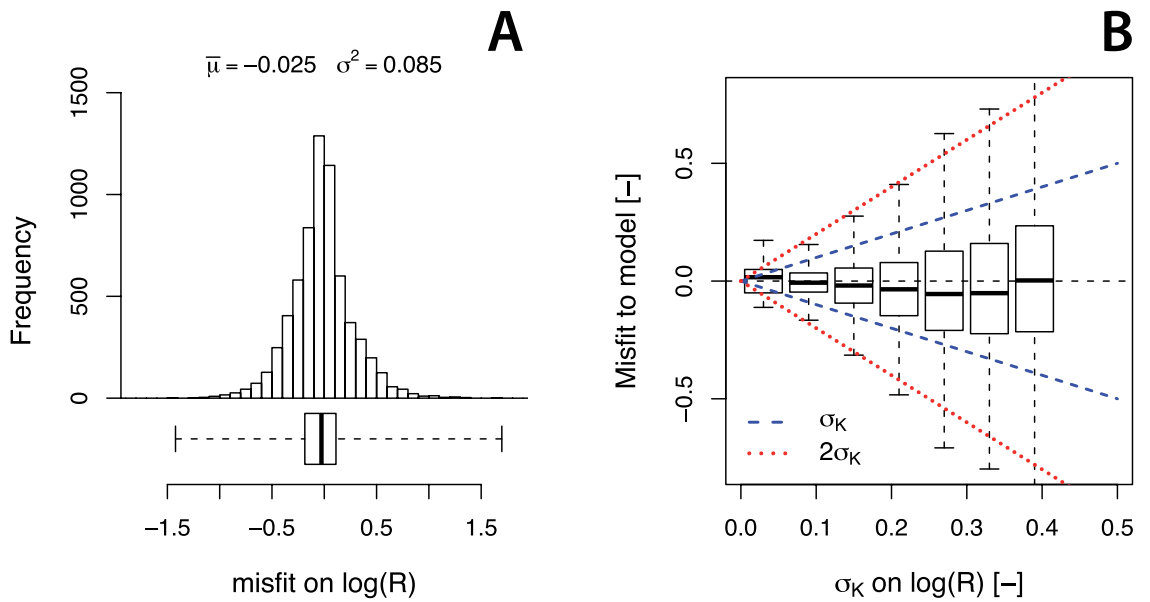

Figure 5: Analysis of misfit between original models and interpolated from S1 subset over S2 area using resistivity of the 1st layer as an example. (A) Misfit has a mean close to zero and standard deviation close to the average value of $\sigma_{K}$. (B) Misfits increase with distance to inversion models and remains within about $\pm \sigma_{K}$ at $68 \%$.

ties in S2 have a mean close to zero and a variance of $\sigma^{2}=0.08$, which is close to the average prediction variances on this parameter $\sigma_{K R I}^{2}=0.1$. In Fig. 5 (B), the misfit logically increases with kriging variances (i.e., with distance to data points in S1), but remains within the $\pm \sigma_{K R I}$ confidence interval at $68 \%$. This means not only that prediction is relatively accurate, but as well that the estimation of uncertainty provided by kriging is reliable on this dataset. The behavior is similar for all other parameters of this model without marked differences. Yet, prediction performs better for layers where the distribution of resistivity is closer to log-normal. Finally, we tested that inverting the two subsets S1 and S2 had no significant effect on the preceding conclusions. As a consequence, the interpolation method can be considered as applicable on this dataset. After kriging of parameters with the whole dataset, the agreement between interpolated 2D matrices of model parameters and 1D inversion models is quantified. For this dataset interpolated at $30 \mathrm{~m}$ resolution, averaged RMSE of log-transformed resistivity is 0.06 , corresponding to an error factor of 1.06 for resistivity, which is acceptable. 


\subsection{Management of uncertainties}

Once the model is built, the analysis of uncertainties away from data (i.e. between flight-lines) is made possible from the prediction of standard deviation $\sigma_{K R I}$, available at each cell of the 2D matrices (Fig. 6, A). As expected, the kriging standard deviation increases with the distance to flight lines. It is shown that in this context, values of log-transformed resistivity, interpolated between the $250 \mathrm{~m}$-spaced flight lines, have a kriging standard deviation of about 0.1 . When combining interpolation uncertainty with inversion uncertainty (Fig. 6, B), the uncertainty increases but remains within an acceptable range, with a total standard deviation of about 0.2 $(\mathrm{STDF}=1.2)$.

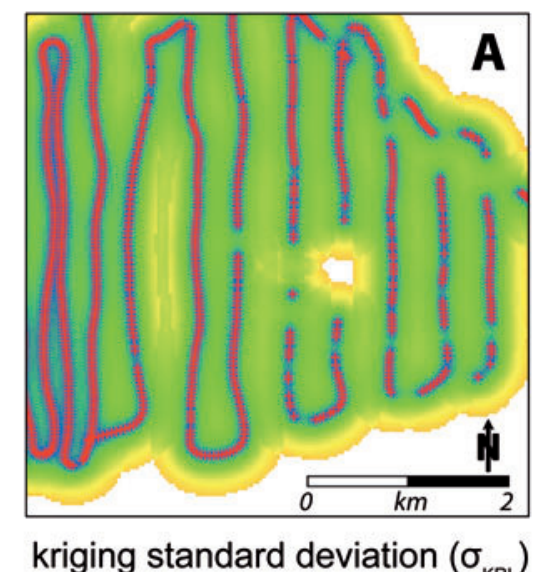

kriging standard deviation $\left(\sigma_{\mathrm{KRI}}\right)$

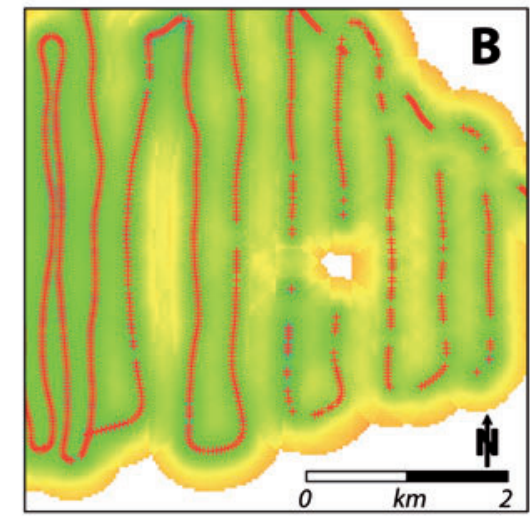

total standard deviation $\left(\sigma_{\text {TOT }}\right)$

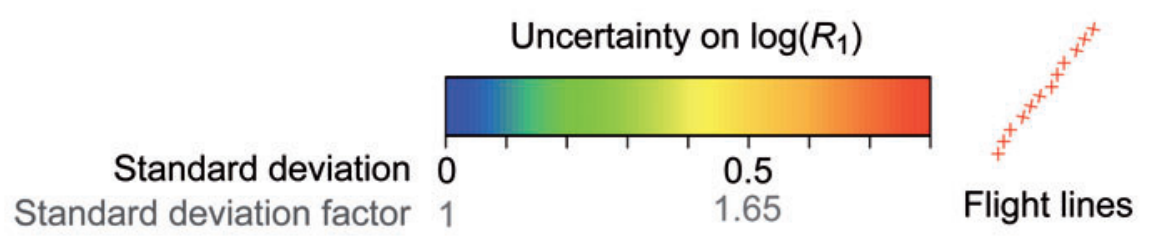

Figure 6: Uncertainty on log-transformed resistivity of the first layer. The area corresponds to the yellow perimeter outlined on Fig. 3. Left (A): kriging standard deviation $\sigma_{K R I}$ increases away from flight lines (red crosses). Right (B): the total standard deviation $\sigma_{T O T}$ combines uncertainties from inversion and kriging.

These results were presented for the 1st layer of the geophysical model. Conclusions remain roughly the same for other layers, at the exception of deeper layers where an increase of kriging standard deviation is observed. 
This loss of accuracy is due to sharp resistivity contrasts for these layers, which is a consequence of sea water intrusion. Yet, larger misfit on resistivity is restricted to a fringe located at the end of the area of detection of sea water intrusion.

\subsection{D Visualization}

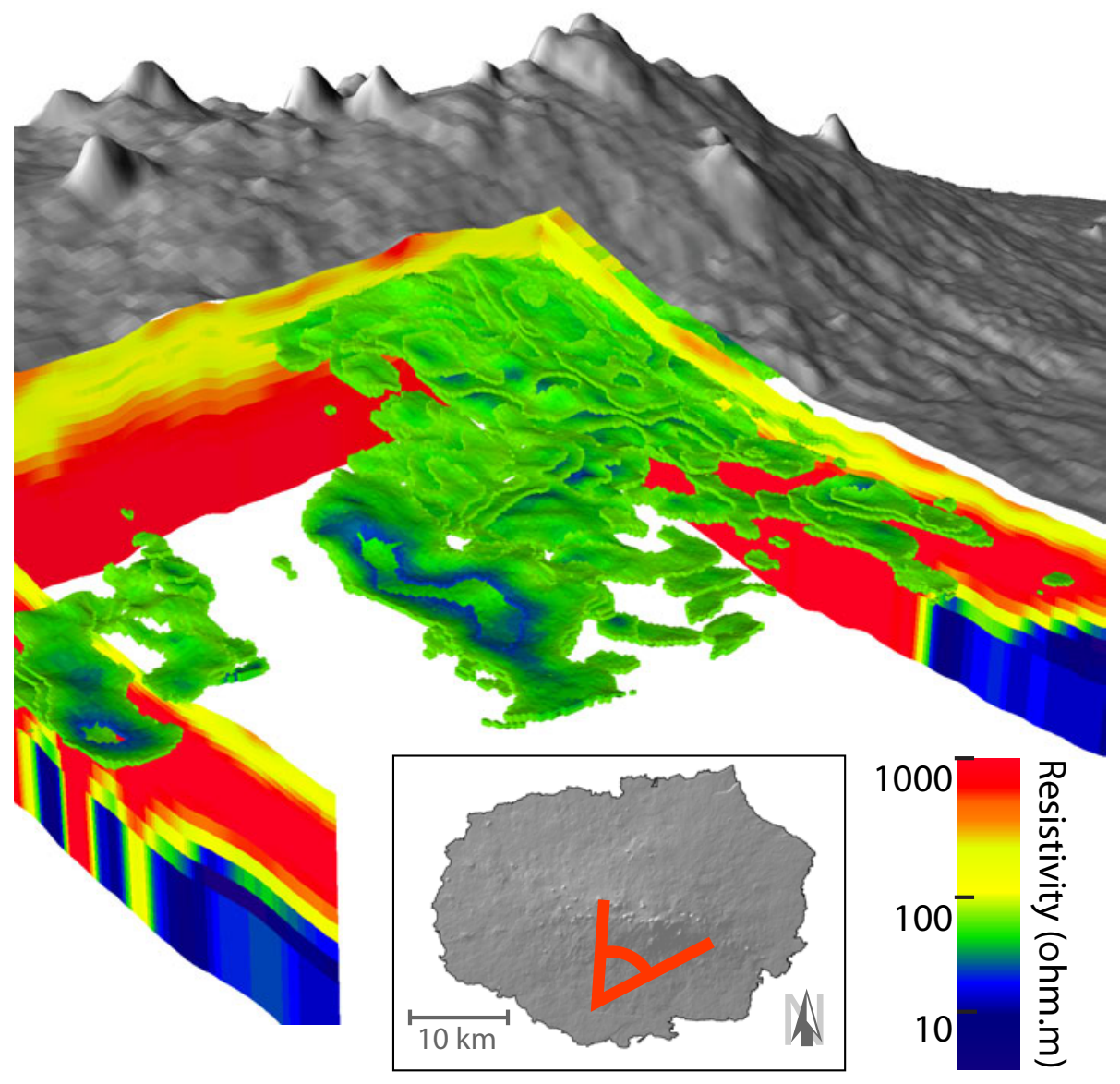

Figure 7: 3D view of SkyTEM survey on Santa Cruz Island reveals the geometry of a large $6 \mathrm{~km}^{3}$ low-resistivity formation, extracted with a 30-70 ohm.m threshold on resistivity values and draped by a high resolution DEM (d'Ozouville et al., 2008b). The red line on inset shows the location and orientation of the virtual camera.

The 3D grid is exported into binary VTK file format, which allows a flexible visualization in VTK-compatible software such as Paraview @R 


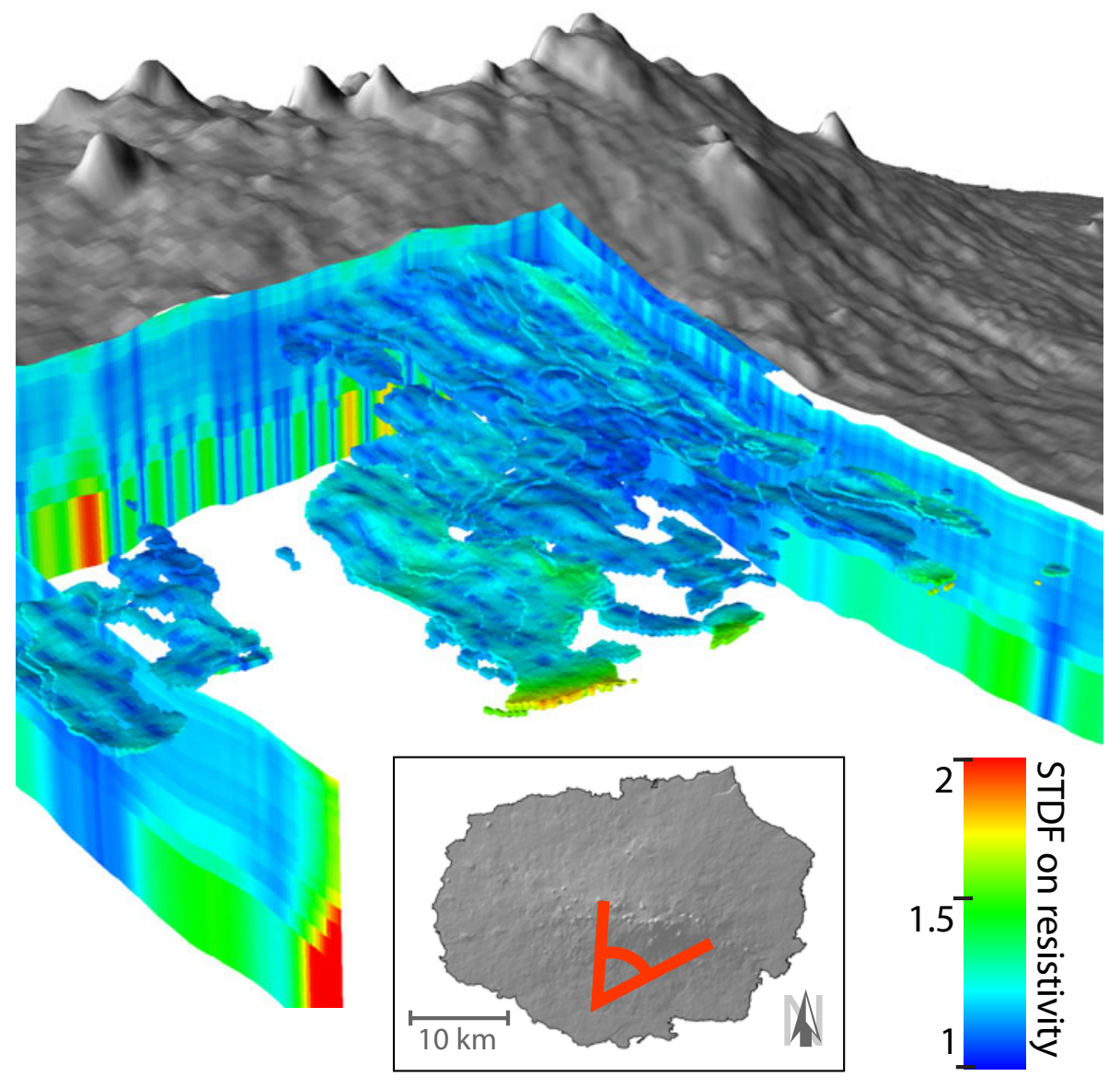

Figure 8: 3D view of total uncertainty on resistivity, integrating error from both inversion and interpolation processes, and expressed as standard deviation factor (STDF). In the background low uncertainty traces (dark-blue) are the imprints of flight lines. The low resistivity formation extracted from resistivity threshold is well resolved. The red line on inset shows the location and orientation of the virtual camera. 
(www.paraview.org). The VTK file containing all geometry and data (resistivity, uncertainties on resistivity and thickness) is easily loadable on a standard machine (tested on $2.4 \mathrm{GHz}$ Intel Core 2 Duo $\AA$, with 4GB RAM). Fig. 7 shows the combined 3D view of "classic" cross-sections, together with a subset of cells extracted by a threshold on resistivity between 30 and $70 \mathrm{ohm} . \mathrm{m}$, and covered by the shaded relief map of a high-resolution DEM (d'Ozouville et al., 2008b). This image highlights the 3D geometry of a large low-resistivity body, first identified by (d'Ozouville et al., 2008a). This feature covers about $50 \mathrm{~km}^{2}$ and appears to be relatively continuous, with a total volume of $6 \mathrm{~km}^{3}$ and a mean thickness of about a $30 \mathrm{~m}$. The 3D map of total uncertainty on resistivity (Fig. 8), expressed as standard deviation factor, illustrates that this feature is well-resolved, with a mean STDF of 1.2. Although the execution of exploration drill holes is still missing to validate this hypothesis, available climatic and geological data can be compatible with the existence of a water saturated and potentially clayey formation, which could fit in the resistivity range of this feature.

\section{Conclusion}

To date, most airborne AEM datasets are inverted with a 1D model description and most of them are visualized as 2D interpolated maps. Numerous extensive AEM datasets have been collected in various 3D geological contexts. They have proven to perform successfully as long as 3D heterogeneities in the subsurface are bigger than the footprint of the soundings. The methodology presented in this paper allows 3D visualization of inversion models and volumetric computations. The $2 \mathrm{D}$ interpolation by kriging of the model parameters is based on the "layered-earth approach". It insures a good coherence with 1D models and conserves the vertical resolution of the inversion, while providing fast grid generation and lightweight files. The quantification of errors combines uncertainties from interpolation and inversion. Using a volcanic case-study, we showed that for a flight line spacing of $250 \mathrm{~m}$, the total uncertainty remains within an acceptable range. However, the uncertainty may increase with larger line spacing and sharp contrasts in resistivity. Because the method is fast and simple, a 3D grid of resistivity can be easily built from extensive surveys covering large scale 3D geological structures. Visualization options include thresholding of resistivity and uncertainty, allowing the user to extract different 3D geological bodies based on resistivity ranges and conceal the data with high uncertainty. This is a step toward enhanced interpretation of AEM datasets. 


\section{Acknowledgements}

This work has been funded by the French Agence Nationale de la Recherche (ANR-blanc 2010 GIIWS $n^{\circ}$ 601-01). The Charles Darwin Research Station and the Galapagos National Park collaborated to the 2006 SkyTEM survey in Galápagos, which was funded by Foundation de France, Foundation Véolia Environnement, Fondation Schlumberger-SEED, Chancellerie des Universités de Paris, UGAFIP-BID and Municipality of Santa Cruz. 


\section{References}

Auken, E., Christiansen, A., 2004. Layered and laterally constrained 2D inversion of resistivity data. Geophysics $69(3), 752-761$.

Auken, E., Christiansen, A.V., Jacobsen, L.H., Sorensen, K.I., 2008. A resolution study of buried valleys using laterally constrained inversion of TEM data. Journal of Applied Geophysics 65(1), 10-20.

Auken, E., Violette, S., d'Ozouville, N., Deffontaines, B., I. Sørensen, K., Viezzoli, A., de Marsily, G., 2009. An integrated study of the hydrogeology of volcanic islands using helicopter borne transient electromagnetic: Application in the Galapagos Archipelago. C. R. Geoscience 341, 899-907.

Bosch, J., Bakker, M.J., Gunnink, J., Paap, B., 2009. Airborne electromagnetic measurements as basis for a 3D geological model of an Elsterian incision. Zeitschrift der Deutschen Gesellschaften für Geowissenschaften 160, 249-258.

Bow, C.S., 1979. The Geology and Petrogenesis of the Lavas of Floreana and Santa Cruz Islands: Galapagos Archipelago. Ph.D. thesis. University of Oregon, USA.

Chilès, J., Delfiner, P., 2011. Geostatistics: Modeling Spatial Uncertainty second ed. Wiley, New York.

Cox, L., Wilson, G., Zhdanov, M., 2010. 3D inversion of airborne electromagnetic data using a moving footprint. Exploration Geophysics 41, 250-259.

d'Ozouville, N., Auken, E., Sorensen, K., Violette, S., de Marsily, G., Deffontaines, B. Merlen, G., 2008a. Extensive perched aquifer and structural implications revealed by $3 \mathrm{D}$ resistivity mapping in a Galapagos volcano. Earth and Planetary Science Letters $269,517-521$.

d'Ozouville, N., Deffontaines, B., Benveniste, J., Wegmüller, U., Violette, S., de Marsily, G., 2008b. DEM generation using ASAR (ENVISAT) for addressing the lack of freshwater ecosystems management, Santa Cruz Island, Galapagos. Remote Sensing of Environment 112, 4131-4147.

Mogi, T., Kusunoki, K., Kaieda, H., Ito, H., Jomori, A., Jomori, N., Yuuki, Y., 2009. Grounded electrical-source airborne transient electromagnetic (GREATEM) survey of Mount Bandai, north-eastern Japan. Exploration Geophysics 40, 1-7 Sp.Iss. SI.

Mullen, I., Kellett, J., 2007. Groundwater salinity mapping using airborne electromagnetics and borehole data within the lower Balonne catchment, Queensland, Australia. International journal of applied earth observation and geoinformation 9, 116-123.

Newman, G., Hohmann, G., Anderson, W., 1986. Transient electromagnetic response of a 3-dimensional body in layered earth. Geophysics 51, 1608-1627.

Palamara, D., Boero Rodriguez, V., Kellett, J., Macaulay, S., 2010. Salt mapping in the Lower Macquarie area, Australia, using airborne electromagnetic data. Environmental Earth Sciences 61, 613-623.

Pebesma, E., 2001. Gstat user's manual. Dept. of Physical Geography, Utrecht University, Utrecht, The Netherlands .

Pebesma, E., Wesseling, C., 1998. Gstat: a program for geostatistical modelling, prediction and simulation. Computers \& Geosciences 24, 17-31.

Reid, J., Pfaffling, A., Vrbancich, J., 2006. Airborne electromagnetic footprints in 1D earths. Geophysics 71, G63-G72.

Steuer, A., Siemon, B., Auken, E., 2009. A comparison of helicopter-borne electromag- 
netics in frequency- and time-domain at the Cuxhaven valley in Northern Germany. Journal of Applied Geophysics 67, 194-205.

Supper, R., Motschka, K., Ahl, A., Bauer-Gottwein, P., Gondwe, B., Alonso, G., Romer, A., Ottowitz, D., Kinzelbach, W., 2009. Spatial mapping of submerged cave systems by means of airborne electromagnetics: an emerging technology to support protection of endangered karst aquifers. Near Surface Geophysics 7, 613-627.

Viezzoli, A., Christiansen, A.V., Auken, E., Sorensen, K., 2008. Quasi-3D modeling of airborne TEM data by spatially constrained inversion. Geophysics 73, F105-F113.

Viezzoli, A., Munday, T., Auken, E., Christiansen, A., 2010. Accurate quasi 3D versus practical full 3D inversion of AEM data. Preview , 23-31. 